\title{
The Mental Health Education of Higher Vocational Colleges from the Perspective of Positive Psychology*
}

\author{
Jie Liang \\ Beijing Vocational College of Agriculture \\ Beijing, China 102208
}

\begin{abstract}
Mental health education in higher vocational colleges has long followed the mental health education model of undergraduate students, which does not meet the psychological characteristics of higher vocational students. Higher vocational students have poor academic performance, low self-esteem, and poor self-control. Their ability to "help others" and "help themselves" is not as good as that of undergraduate students. Such a situation requires that the mental health education work of higher vocational colleges should break through the traditional single and limited working mode, and use the theory and methods of positive psychology to awaken the potential positive power of higher vocational students, so as to resist their negative qualities to encourage them to experience happiness and gain growth.
\end{abstract}

Keywords-positive psychology; higher vocational students; mental health

\section{INTRODUCTION}

In 1999, UNESCO pointed out in the "Recommendations of the Second International Conference on Vocational and Technical Education and Training" that "we need to enable more people to receive vocational education, to enable vocational education to meet the needs of talent resource development and to enable people to effectively enter the world of work." With the rapid development of science and technology in the 21 st century, society has higher expectations for colleges in terms of knowledge innovation and personnel training. Schopenhauer said that good conscience is more important than good reputation. Your biggest goal is to be healthy and mentally healthy. In this way, your thinking will not be exhausted, you will be independent and then have a good life. Therefore, higher vocational students who are the main social productive forces have good psychological literacy and maintain a positive and happy attitude, which has positive practical significance for both personal growth and social development.

In recent years, the phenomenon that college students hurt themselves and others due to psychological problems has

*Fund Project: One of the research results of the Teaching Reform Research Project of Beijing Vocational College of Agriculture "Research on the Construction of Positive Personality of Higher Vocational Agricultural Majors from the Perspective of Positive Psychology"

(Approve number: 2017046, Director: Jie Liang). occurred from time to time, which has caused the government and schools to attach great importance to mental health education. The growth environment, psychological characteristics and training objectives of higher vocational students have certain "specificity", so mental health education is particularly important. After the 1990s, various higher vocational colleges have set up mental health education centers, making great progress in mental health education and psychological counseling, but there are still limitations. The research direction is centered on the problem, which makes the mental health research and practice of higher vocational colleges overemphasize the correction of psychological problems and the treatment of mental illnesses. It tends to neglect the mental health education for most students, so that the students' enthusiasm for class is not high, and the interest in learning is not strong. At the same time, the mental health self-education of the students is neglected. Therefore, the school mental health education work urgently needs to change the perspective to stimulate the students' internal positive strength to promote their healthy growth and meet the needs of the society for high-skilled talents. In this context, positive psychology enters the field of vision of the school.

Positive psychology is a brand-new psychological trend that emerged in the United States at the end of the twentieth century. It abandons the excessive attention of traditional psychology in the prevention and treatment of mental illness. It advocates looking at individuals with a positive eye, believing that everyone has positive energy and potential virtues and they can use these positive forces and qualities to overcome their own psychological and behavioral problems. Only when the positive forces inherent in human beings are nurtured and grown can the negative aspects of humanity be eliminated or suppressed, which will contribute to harmonious development. Therefore, higher vocational mental health education should not only study the status quo and causes of students' mental health, but also pay attention to cultivating the positive subjective experience and positive personality traits of higher vocational students and shaping the positive social environment. This is a new perspective and new ideas for the current mental health education work in higher vocational schools. 


\section{Mental Health Status of Higher Vocational STUDENTS}

The 18-25 years old in the higher vocational college stage is "the beginning of the maturity", which is an important stage of life, and also a key period for the formation of personality characteristics, outlook on life and world outlook. Such a specific educational environment and age structure make them develop unique psychological characteristics. Most of the higher vocational students have a lack of self-confidence, a lack of emotional management skills, poor learning adaptability, and low levels of willpower.

\section{A. Mental Health Status of Higher Vocational Students Reflected in Mental Health Census}

Due to the failure of the college entrance examination, the higher vocational students did not enter the ideal university, so they had to choose the higher vocational colleges. Therefore, they feel that they are in a weak position compared with their peers. The society's perception of higher vocational college students affects their sense of identity. Zhang Zhaohe, an associate professor at the Hong Kong University of Science and Technology, believes that "whether a group's identity can be recognized and respected by the society is a very important thing for the group."Psychologist Adler believes that people's inferiority complex is caused by two aspects. One is caused by neglect or refusal. If a person is neglected, it will lead to his loss of self-worth, which will bring him a huge insurmountable inferiority complex. Most students in higher vocational colleges were generally in a lower position when they are in middle school and even in elementary schools, so they cannot get the attention of teachers. They lack a successful experience, and they are always more criticized and accused than being praised and encouraged, thus forming their inferiority. In the mental health classroom, the teacher asked the students to write three successful things. Many students could not write out one, because they thought they had no success, and never had a successful happy experience. Long-term study life forms habitual helplessness, negative self-suggestion, so that they cannot see their own advantages and strengths. Lacking confidence in myself, thinking that their ability is poor, and the ability of communication is not good, which also affects their interpersonal relationship to a certain extent. The tense interpersonal relationship caused by the lack of social intelligence will make them feel weak and cause a low sense of self-worth.

1) Negative personality trait: Higher vocational students are in three states when they are in class: a small number of students will actively participate, and they can follow the teacher's positive thinking and take the initiative to answer questions; Some students are at the edge, that is, sometimes they look up and take notes, sometimes they are in a state of emptying their minds;most of them are in a state of not attending classes or sleeping, don't take notes, and don't practice. Although most of the students do not skip classes, there are not many students who can concentrate on listening to the lectures. Their thinking is in a state of dissociation, and the emotions are indifferent. They have no emotional resonance and behavioral interactions with the teacher's content, and are in a state of nonchalance. There are other students who feel anxious and fearful because they cannot understand the content of learning. They are difficult to adapt to the learning environment created by teachers and become marginal learners. Because long-term study life forms habitual helplessness, their perception of self is "I am stupid, others will laugh at me" "I am not a good student"... If these bad habits continue, it will make them lack the curiosity and interest in exploring new things. Even if they are faced with some simple problems, they are not willing to do it, because they are afraid of facing the wrongand are not willing to face the "facts that I will make mistakes." So they chose to delay the face, and ultimately their sense of self-efficacy is reduced, and creativity is impossible to talk about.

2) Lack of restraint and poor self-control: Previous studies have shown that there is a high correlation between learning self-control and academic achievement, and the former has a good predictive power for the latter. Under normal circumstances, the academic performance of students with high self-control is better than that of students with relatively weak self-control. The long-term academic disability of students in higher vocational colleges is largely related to their poor self-control. The specific performance is that there is no good study habit. For example, active inquiry, doubt, reflection. Lack of tenacity of will, unable to control their own behavior, one of the performances is to avoid difficulties, cannot bear hardships and endurance. Lack of social responsibility and collective sense of honor, higher vocational students are in the process of growth, the impact of mainstream culture, the drive of utilitarian thoughts, the alienation of social emotions, making personal self-interested thinking serious, and the assignments are delayed until the last minute. Overdraft parents give pocket money; play online games all night to relax themselves; always want to lose weight, but can't stick to it. Some students have not yet fully developed the central nervous system, and the nerve fibers have not been completely myelination. The transmitted nerve impulses are easy to generalize and are not accurate enough. Therefore, their self-control is relatively poor. They are extremely emotional, impulsive, and conflict with classmates, teachers, and parents. One manifestation of poor behavioral self-control is the use of mobile phone addiction. In the interview, it was found that many mobile phones for higher vocational students have replaced computers. Mobile phones are not only convenient but also concealed, and are less likely to be discovered by teachers and parents. Some students have a strong desire to play mobile phones, and they cannot concentrate on class. Once the mobile phone is taken away, those students will have withdrawal or physical symptoms.

\section{B. Student Case Consultation and Analysis in Higher Vocational Colleges}

The main content of the students' consultation is life and learning adaptation problems, emotional problems (mainly about heterosexual communication), interpersonal relationship problems, learning problems, and employment pressure issues. 
Since the establishment of the consultation center, the abovementioned consultation content has remained basically the same, but the depth is different, especially the emotional relationship problem.

1) Interpersonal relationship problem: Case: Wang, female, 16 years old, first-year accounting major. She lives with her grandparents and is spoiled and willful. When she is at school, she postpones doing something and slows down. Thus everyone starts waiting for her and slowly alienates her. She only has a good relationship with a girl in the class, but she sometimes doesn't like her and gets mad at her. Afterwards, she apologized to the girl for fear of loneliness, but at that time the girl was fed up (crying) and emotional.

The interpersonal relationship problem of higher vocational students is mainly between roommates or classmates. Some individual students may have tensions or contradictions in their relationships because of their self-centeredness, inferiority, despondence, and other personal reasons, or because of differences in values and living habits. Also the separation of some students from their good friends by division of classes or dormitories will cause their emotional distress. The reason for this is because most students generally lack the sense of cooperation based on equal respect and lack of methods to resolve interpersonal disputes. Introverted children are more vulnerable to interpersonal relationships.

2) Adaptation problem: Case: Zhou, female, 18 years old. Her parents are farmers, her family has a good economic situation, her academic performance is medium, and her personality is introverted. Going to higher vocational colleges is the first time she left her family to live a collective life. In the past few months, she always felt that it was difficult to adapt to the school's learning and living environment. Even in the crowd, she felt that her heart was empty. If she hears the laughter of her classmates, she will feel that she is left out. She often cries because of her homesickness. She will be restless every Thursday, and she has the idea of transferring to another school or dropping out of school several times. In the classroom, her attention was difficult to concentrate, so her academic performance declined, and her heart was in a mess.

New students in higher vocational colleges will not be able to adapt to the new environment. Because most of the students left home to come to a new environment and live with strangers of similar age for the first time, it is normal to have psychological discomfort. Loneliness and feelings of loss to the school are obvious manifestations of higher vocational students. Some students can adjust themselves very quickly, but the introverted classmates have less ability to adjust. If some students can't adjust themselves, this will cause obstacles to their ability to adapt.

3) Emotional problem: Case: Wang, male, 20 years old, junior student of the Automobile Maintenance major. He has antagonistic feelings and his mood is fluctuating. He said that his personality is introverted, and he never shared his heart to others, including parents. He started to live in school from elementary school, and later studied wrestling at the sports school, but his performance was not good. In adolescence, he often lost his temper because of trivial matters. He was easily provoked.He often had physical conflicts with other students because of the problem of heterosexual relationships, but he wouldfeel sorry about that afterwards. A female student once said that she had lost 12 pounds in 6 days after breaking up with her boyfriend who had been in contact for 9 months. It was difficult for her to extricate herself and the idea of dropping out of school came up to her.

Students' emotional ups and downs during adolescence, self-conscious students will be confused because they are too concerned about their emotions, which will strengthen their negative emotions. Adolescent students have a tentative and transient nature of their exploration of love. At this time, they are thinking more about who can bring more happy moments to them. Therefore, students are too much in the pursuit of psychological experience and emotional needs, regardless of the consequences. If they put too much emotion into their interactions, they will be indecisive when they encounter setbacks or conflicts, and tend to suffer deeper damage, so that they need to adjust themselves for a long time to return to normal. Because of the birth of their sexual psychology, and the psychological development is not mature, the students show great blindness and impulsiveness in dealing with emotional issues.

4) Learning problem: Case: Wang, his self-control ability is very poor. The main performance is that he was obsessed with online games in the third grade of junior high school. It is useless for parents to educate him. He does nothing after school every day, and then plays games until midnight.Later, he suddenly felt that playing the game was boring and wanted to study hard at this school. However, because he couldn't concentrate and his memory was not good, he couldn't keep upin many subjects. Then he gradually lost interest and felt bored.

Higher vocational students have poor self-control ability and are easily influenced by others. Because of the conformity and the pursuit of adulthood, they have the idea that "other people fall in love, I also want to fall in love" "other people play games, I also play games", they will not be able to extricate themselves over time. The underlying reason for their lack of motivation to learning is the lack of lofty ideals and the lack of a correct outlook on life. A lot of students don't know what they are living for, and why they go to school, etc. If they don't clarify these fundamental problems, they can't solve their problem of lack of learning motivation.

Based on the psychological characteristics of students in higher vocational colleges, we must break through the limitations of traditional education in the mental health education of schools. According to the students' inherent positive ability and positive potential, we will strive to create a mental health education model suitable for the growth of higher vocational students, paying attention to the positive personality quality characteristics of students, stimulating and enhancing their positive personality quality, and giving full play to their inherent positive advantages to promote their healthy and harmonious growth. 


\section{THE REALIZATION OF THE Positive DEVELOPMENT OF MENTAL HEALTH EdUCATION IN HighER VocationaL COLLEGES}

The maintenance of students' mental health requires all parties to cooperate and actively promote the mental health development of all students, prevent their mental illness, and solve their psychological distress and obstacles, so as to meet professional needs and personal health growth needs, and ultimately promote social harmony. Therefore, the mental health education work of higher vocational colleges should use positive psychology as the theoretical framework to design practical activities to stimulate students' positive emotions, cultivate their positive personality, and realize the positive development of mental health education in higher vocational colleges.

\section{A. Positive Development of Teaching Methods}

Students in higher vocational colleges have strong practical ability, while it is difficult for them to accept theoretical knowledge. In this way, the mental health curriculum of higher vocational colleges should be freed from the old frame of "teacher-centered, textbook-centered, classroom-centered", truly establish the teaching philosophy of "student-centered, learn-to-use-centered" and focus on the active participation of all students. Teachers are no longer the protagonists of the classroom, but the organizers and designers of the classroom, the guides of the classroom process, and strive to design each psychological lesson. Teachers should try their best to focus on activities, mainly on student experience, and use psychological tests, videos, music, psychological dramas, discussions and other means in the psychological education classroom. The effect of this is to enable each student to have the opportunity to speak in the classroom, and to participate in the classroom activities, so that all students are involved. Through enthusiastic activities, students' emotions can be experienced, behaviors can be trained. At the same time, their mutual cooperation and mutual communication in the activities have enhanced the interpersonal trust and cooperation concept among the students, which makes the classroom environment more harmonious and the classroom effect more obvious.

\section{B. Focusing on Cultivating Optimism in Individual Counseling}

Positive psychology believes that positive emotions include happiness, interest, satisfaction, pride, and love. These can help individuals break through limitations and develop experiences, expand their ability to recognize cognitive behaviors instantaneously, and build and enhance their personal capital. It is true that there are various causes for the psychological problems of students in higher vocational colleges. However, if we only entangle the root causes of the problems in the consultation, it does not help the students. Therefore, in individual counseling, we should pay more attention to cultivating students' positive emotions, such as optimism. We subjectively believe that many students in higher vocational colleges have intrinsic learning disabilities that cannot be changed. However, it turns out that many of these psychological disorders are just their aversion to learning, that is, because of the improper learning methods, students can't improve their academic performance no matter how hard they work. Since the efforts have proven to be ineffective for a long time, their motivation for learning will decline and then feel bored with learning. This is what psychologist Seligman calls "learned helplessness." Seligman believes that since helplessness is learned, positive and optimistic emotions should also be learned. In particular, the rise of positive psychology has led people to turn their attention to the acquisition of positive resources. Optimism as a kind of psychological capital or positive psychological power can be obtained through a certain psychological mechanism with the help of teachers or adults, through their own hard work and learning. Combined with cognitive therapy technology, Seligman proposed a method to develop students' positive emotions, called ABCDE technology. When an unpleasant incident occurs, it is natural for us to think about it and quickly develop some thoughts. These thoughts will become very accustomed, so that we will unconsciously think like this, and then these thoughts will cause consequences, so what we do is the direct consequence of these thoughts. It is the key to our abandonment and loss, and also the key to cheer up and try again. D means disprove and $\mathrm{E}$ means encourage. Training with this method regularly, students will not give up or feel pessimistic and helpless even if they encounter difficulties in their studies. Teachers and parents should also learn to be optimistic about cognitive skills and incorporate it into their own way of thinking to guide students.

\section{Creating a Positive Social Environment}

From the perspective of the ecosystem, people's psychological activities have corresponding cultural backgrounds, based on various activities in the environmental system. Only changing the student factor is not able to save them from the work dilemma. The "Ecological Systems Theory" proposed by American psychologist Bronfenbrenner divides the relationship between human and environment into microsystems, medium systems, external systems and macrosystems according to their closeness. Microsystems are the closest external environment to individuals, studying the relationship between people and the environment, emphasizing the role of objectivity in subjectivity.

1) Teachers use emotion as the center to create a good classroom teaching environment: Teacher-student relationship is a partnership. In the teaching of mental health curriculum, teachers should look at the whole world from the standpoint of students to create an atmosphere of communicationwith empathy. Fromm's theory of needs tells us that people have their own needs. People need to communicate with others, to exchange informationand emotional thoughts through various forms such as communication and participation activities. Therefore, the indifference of students in the classroom is actually a defensive reaction, and such a state of dissociation is usually associated with a wrong environment that obstructs their needs. The traditional classroom is that the teachersalways speak and speak and they regard students as the same individual to control the whole. Such one-way classrooms make the teachers and students lack emotional communication 
and communication. Teaching is a manifestation of relationship, a dialogue between teachers and students, but not a unilateral subject behavior. Classroom situations are generated through interactive communication and dialogue between teachers and students. The establishment of an intimate relationship between teachers and students can give students the opportunity to face and heal their fault, thus changing the wrong ideas stemming from the pain. If the teacher respects, empathizes, and understands the student, the student will experience a change in mental function and become more sensitive, active, and initiative. Creating a fair and equitable learning atmosphere allows students to believe that his efforts can be seen by teachers regardless of his grades.

2) Parents focus on encouragement and appreciation to create a good family environment: Many Chinese are very inferior, especially for high-school students. This is because our parents often do not respect their children. How can people who grow up in a culture of lack of respect not feel inferior? Family is the place where children begin to construct their convictions, and it is also the place where children first feel emotions. The two most important points for children are "security" and "I am important". The lacks of both will fundamentally cause their neurotic personality when they grow up. There's nothing wrong with thatmost parents hope their children will have a bright future. However, most parents are concerned about their children's academic performance, rather than caring about their emotional and health problems. Some people have conducted surveys, which show that Chinese parents' attention rate on learning and emotional issues can reach $64 \%$ to $1 \%$. So, from the perspective of life, is the importance of academic achievement is really more than 60 times of the importance of physical health and emotional stability? Parents should understand their children and cannot expect them too much. This will go beyond the child's inner strength and beyond his ability, so that parents' expectations will become the source of their anxiety. According to Vygotsky'sZone of Proximal Development, parents should set reasonable goals based on children's ability and level. If the goals are too low, they will feel unconquered. Encouraging easy success will reduce children's self-esteem and will cost them a heavy price in their future education. Parents should reward their children in a timely manner, and encouragement should focus on the process rather than the outcome.

3) Schools should rely on and shape a good environment to achieve ecological education: Marx said that people create the environment, and the same, the environment also creates people. Ecological psychology believes that people and the environment interact to each other, and they are integrated, and education pursues the organic harmony between people and the environment. Therefore, the natural environment and social environment are important resources for schools to carry out mental health education. The mental health education in higher vocational colleges should break through the limitations of the original mental health education work, using the ecological environment resources to innovate a new mental health education model, and using ecological psychological counseling as the teaching content, particularly using natural environmental resources and humanistic environment to develop psychological counseling methods, such as forest therapy, horticultural therapy, wilderness therapy, etc. to improve students' mental health; students can experience better and more positive emotions in a good natural environment, learn to love, awe and gratitude nature, and cultivate positive personality.

\section{CONCLUSION}

Students in higher vocational colleges are the pillars of the motherland and the builders and main force of the future society. Their psychological quality directly affects the development of various undertakings in China and the harmony and stability of the future society. The mental health education work in higher vocational colleges has a long way to go. The positive psychology provides a new perspective for mental health education in higher vocational colleges and will have a far-reaching impact on the future.

\section{REFERENCES}

[1] Peterson, C., Seligman, M. E. P. Character Strengths andVirtues: A Handbook and Classification [M]. New York: OxfordUniversity Press, 2004.

[2] Bandura. "Social Foundations of Thought and Action: A Social Cognitive Theory" $[\mathrm{M}]$. Lin Ying, translated. Shanghai: East China Normal University Press, 2007: 55-56. (in Chinese) 